\title{
The influence of dialect in sound symbolic size perception
}

\author{
Andrew Shibata*
}

\begin{abstract}
Prior research on sound symbolism and referent object size establishes that words with front vowels are perceived to refer to smaller objects than do back vowels (Ohala 1997; Klink 2000). Some dialects of American English exhibit vowel movement along the front-back axis which may influence perceived object size. This study focuses on California English /u/-fronting (Hinton et al. 1987) and predicts that shifting from a standardly back vowel $[\mathrm{u}]$ to a more front vowel $[\mathrm{H}]$ is paired with a shift from a large perceived object size to a smaller perceived object size. This paper describes two experiments in which participants either silently read (reading task) or listened (listening task) to stimulus words and rated perceived object size. California English speakers in the reading task experiment perceived words with /u/ to be smaller than did non-California English speakers. This result suggests that sound symbolic perception is sensitive to fine phonetic variability due to a person's dialect.
\end{abstract}

Keywords. sound symbolism; California English; dialect; sociophonetics; perception

1. Introduction. One long-held assumption in linguistics is that the physical form of a linguistic signal is independent of its meaning. This idea is attributed to Ferdinand de Saussure who stated in his Course in General Linguistics that "the bond between the signifier and the signified is arbitrary" (de Saussure 1916, transl. 2011). This arbitrariness principle holds for the majority of lexical items across languages, but Saussure acknowledges that some forms of sound symbolism, such as onomatopoeia, are problematic for a strong view of arbitrariness. While there has been sound symbolic research at the word level (onomatopoeia; Bredin 1996) and morpheme level (phonaesthemes; Bergen 2004), this study uses the term 'sound symbolism' to refer to semantic information conveyed at the individual sound segment level.

In 1997, Ohala defined sound symbolism as the "hypothesized systematic relationship between sound and meaning". He presented cross-linguistic examples from languages such as Ewe, Yoruba, Spanish, English, and French demonstrating that words for "small" seem to contain [i] and [e] vowels while words for "large" seem to contain [a] or [o]. Additional sound symbolism research finds similar binary directional relationships in domains such as perceived texture (Köhler 1929; Ramachandran \& Hubbard 2001), temperature, gender, and weight (Klink 2000). These directions appear to be consistent across languages, inviting research into which properties of speech sounds contribute meaning.

Posited phonetic motivations for sound symbolic mappings include meaning being associated with facial expressions and intrinsic vowel pitch. Ohala (1997) argues that facial expressions conveying emotion in parallel with speech production may explain sound symbolic directions. For example, smiling draws the lips back and effectively shortens the vocal tract, resulting in higher frequency productions. Modulating articulatory features such as lip rounding may lead to associations between friendly or innocuous objects with higher frequency sounds as

\footnotetext{
* This work was completed as undergraduate honors research at UC Berkeley. Special thanks to Keith Johnson, Susan Lin, and Alice Shen for study design and analysis; Daniel Pok for stimulus design; Marla Johnson and Samy Abdel-gaffir for modeling assistance; Sarah Bakst, Sahar Yousef, Michael Silver, Claudia Valdivia, James Michaelov, and Florine Pascal for writing review. Author: Andrew Shibata (andrewshibata@gmail.com).
} 
well as aggressive or larger objects with lower frequency sounds. Another hypothesis, intrinsic vowel pitch, argues that high vowels exhibit higher average F0 values compared to low vowels (Whalen \& Levitt 1995). Analogously with the facial expressions narrative, high vowels can be associated with smaller objects and low vowels can be associated with larger objects.

While much work has been done to establish the existence of sound symbolic domain mappings in extreme polar directions, there is a gap in research exploring to what degree sound symbolic mappings are continuous along vowel height and frontness dimensions. One source of phonetic variability along the same dimensions that sound symbolism research surveys is regional vowel shifts characteristic of speech dialect (Labov, Ash, \& Boberg 2006). Perhaps sound symbolic perception is sensitive the phonetic properties of a person's dialect and changes in agreement with their dialect's vowel shift directions. This current study investigates the relationship between sound symbolic perception and phonetic variation due to dialect properties.

2. Background. The specific sound symbolic domain that this study examines is referent object size. Prior research on sound symbolism and size consistently finds that words containing low vowels are perceived to refer to larger objects compared to high vowels (Sapir 1929; Shinohara \& Kawahara 2010) and back vowels are perceived to refer to larger objects compared to front vowels (Ohala 1997; Klink 2000). These directions are indicated by the outer arrows in Figure 1.

The dialect feature that this study focuses on is / $\mathrm{u}$-fronting in California (CA) English. One characteristic phonetic feature of the CA English dialect is that speakers often produce $/ \mathrm{u} /$ as more fronted compared to General American English (Hinton et al. 1987).

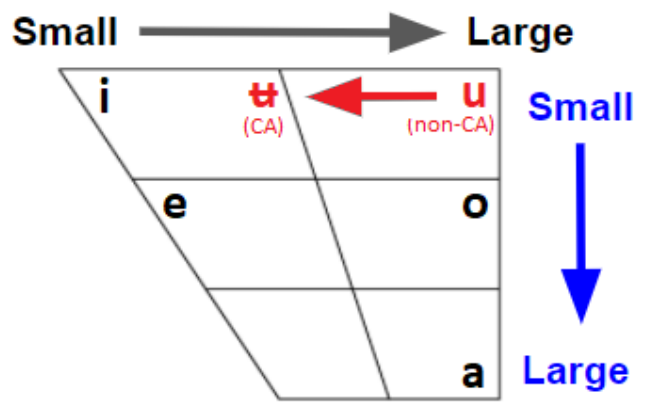

Figure 1. Directions for sound symbolic size perception and $[\mathrm{u}] \rightarrow[\mathrm{u}]$ vowel shift in CA English

Since front vowels are associated with a small perceived object size and /u/-fronting involves shifting a standardly back vowel forward, a continuous symbolic perception hypothesis predicts that /u/-fronting in CA English shifts perceived referent object size for words containing $\mathrm{l} / \mathrm{f}$ from a larger size to a smaller size. To explore this hypothesis, this study involves two experiments comparing perceived referent object size between CA English speakers and non-CA English speakers.

3. Experiments. The general experimental procedure was modeled after Shinohara \& Kawahara (2010). Participants rated the perceived object size of V.CVC nonce words (shown in Table 1) on a Likert scale from 1-4 (Very Small to Very Large). Participants responded to the following prompt from Shinohara \& Kawahara:

Imagine an exotic language that you don't know. The language has a rich lexical inventory of adjectives that express a variety of "largeness" or "smallness". Now, a speaker of this language looks inside a box and finds a jewel. She verbally expresses how 
large or small it looks using one of these adjectives. Your task is to read each of the following words and guess its meaning - i.e., how large or small it is.

This study involved two experiments: a reading task group in which participants silently read the stimulus words and a listening task in which participants listened to the same list of stimuli. The reading task group included 20 CA English speakers ${ }^{1}$ and 22 non-CA English speakers. The listening task group included a separate set of 20 CA English speakers and 20 nonCA English speakers. 2 participants from the reading task group were excluded due to not being native English speakers ${ }^{2}$ and 5 participants from the listening task group were excluded due to not being native English speakers. All participants were UC Berkeley affiliated volunteers.

\begin{tabular}{|c|c|c|c|c|c|c|c|c|c|}
\hline & & \multicolumn{4}{|c|}{ Voiceless Consonants } & \multicolumn{4}{c|}{ Voiced Consonants } \\
\hline \multirow{4}{*}{} & & $\mathbf{p}$ & $\mathbf{t}$ & $\mathbf{S}$ & $\mathbf{k}$ & $\mathbf{b}$ & $\mathbf{d}$ & $\mathbf{z}$ & $\mathbf{g}$ \\
\cline { 2 - 10 } & $\mathbf{i}$ & ipip & itit & isis & ikik & ibib & idid & iziz & igig \\
\cline { 2 - 10 } & e & epep & etet & eses & ekek & ebeb & eded & ezez & egeg \\
\cline { 2 - 10 } & a & apap & atat & asas & akak & abab & adad & azaz & agag \\
\cline { 2 - 9 } & o & opop & otot & osos & okok & obob & odod & ozoz & ogog \\
\cline { 2 - 9 } & u & upup & utut & usus & ukuk & ubub & udud & uzuz & ugug \\
\hline
\end{tabular}

Table 1: List of stimulus words

3.1. READING TASK. In the reading task experiment, participants silently read each stimulus word and rated perceived object size. The CA English speakers received a paper questionnaire, following the testing conditions in Shinohara \& Kawahara (2010). For ease of distribution, all other participants completed the task in the form of a Google Form questionnaire.

There was a significant difference in the reading task between CA and non-CA mean perceived size for objects described using words with /u/ as shown in Figure 2 ( $p<0.0001)$.

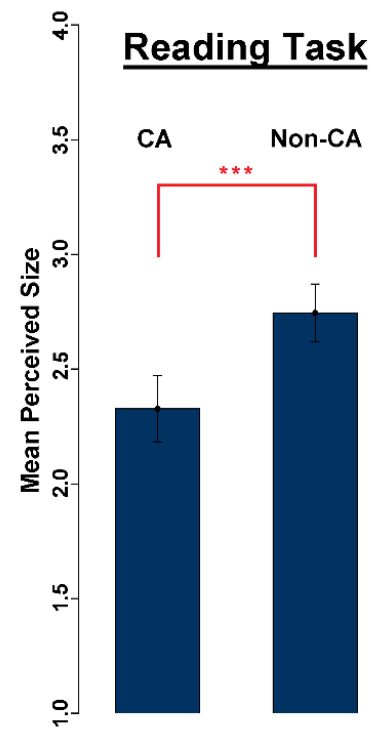

Figure 2. Reading task mean perceived size ratings $\left(\mathrm{S}_{\mathrm{CA}}=2.33, \mathrm{~S}_{\mathrm{non}-\mathrm{CA}}=2.74\right)$

\footnotetext{
${ }^{1}$ Participants were asked in which state they attended high school as an indicator of California dialect membership

${ }^{2}$ For this study, being a native English speaker is defined as having begun the process of learning English by age 3
} 
3.2. LISTENING TASK. In the listening task experiment, participants listened to audio readings of the stimulus word list shown in Table 1 and rated perceived object size on the same Likert scale. This task was delivered as a Google Form questionnaire with the stimuli audio embedded as YouTube videos. Table 2 reports the stimulus speaker's mean formants measured using Praat (2 vowel tokens per word $* 8$ stimulus words per vowel $=16$ instances of each vowel). The stimulus audio recordings did not exhibit significant /u/-fronting. The 3 modifications to the instructions from the reading task were changing the pronoun "she" to "he" to match the gender of the speaker, changing "read" to "listen to" to match the task at hand, and adding the instruction "Please use earphones so that you can hear the words properly" so that the audio could be heard clearly.

\begin{tabular}{|l|c|c|c|c|c|}
\hline Vowel & i & e & a & o & u \\
\hline Mean F1 (Hz) & 262.38 & 387.46 & 734.76 & 462.62 & 302.30 \\
\hline Mean F2 (Hz) & 2326.70 & 2188.01 & 1028.90 & 893.89 & 990.16 \\
\hline
\end{tabular}

Table 2: Mean formant values for listening task speaker

There was not a significant difference in the listening task between CA and non-CA mean perceived size for objects described using words with /u/ as shown in Figure $3(p=0.2421)$.

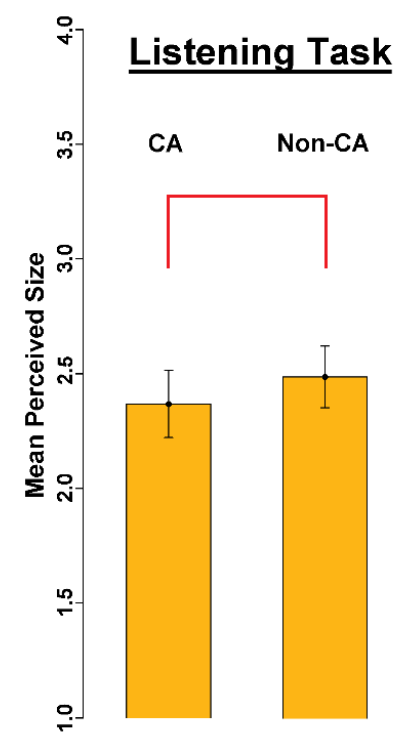

Figure 3. Listening task mean perceived size ratings $\left(\mathrm{S}_{\mathrm{CA}}=2.37, \mathrm{~S}_{\mathrm{non}-\mathrm{CA}}=2.49\right)$

4. Discussion. Phonetic properties of a person's dialect influence perceived referent object size during silent reading. CA English speakers in the reading task rated words with $/ \mathrm{u} /$ as smaller perceived objects compared to non-CA English speakers. This is consistent with the continuous symbolic perception hypothesis that /u/-fronting in CA English shifts perceived referent object size towards a smaller size. Sound symbolic perception appears to be sensitive to fine phonetic information from a speaker's dialect and moves in the same direction as the dialect shift.

The results for the listening task did not find an effect of CA English dialect membership on perceived size. This suggests that people perceive referent object size based on the acoustic properties of the signal they hear. Additionally, since / $\mathrm{u}$ / is both a back vowel (associated with large perceived size) as well as a high vowel (associated with small perceived size), both speaker 
groups perceiving / $\mathrm{u} /$ as moderately sized supports a feature-based approach to sound symbolic perception. Overall sound symbolic perception then results from integrating meaning from multiple articulatory dimensions such as vowel height and vowel frontness.

Finding that phonetic variation influences sound symbolic perception yields implications for the relative ordering of semantic processing and phonological abstraction during on-line speech processing. Figure 4 presents two hypothetical orderings of fine phonetic detail, phonological abstraction, and extracting sound symbolic meaning from a signal.
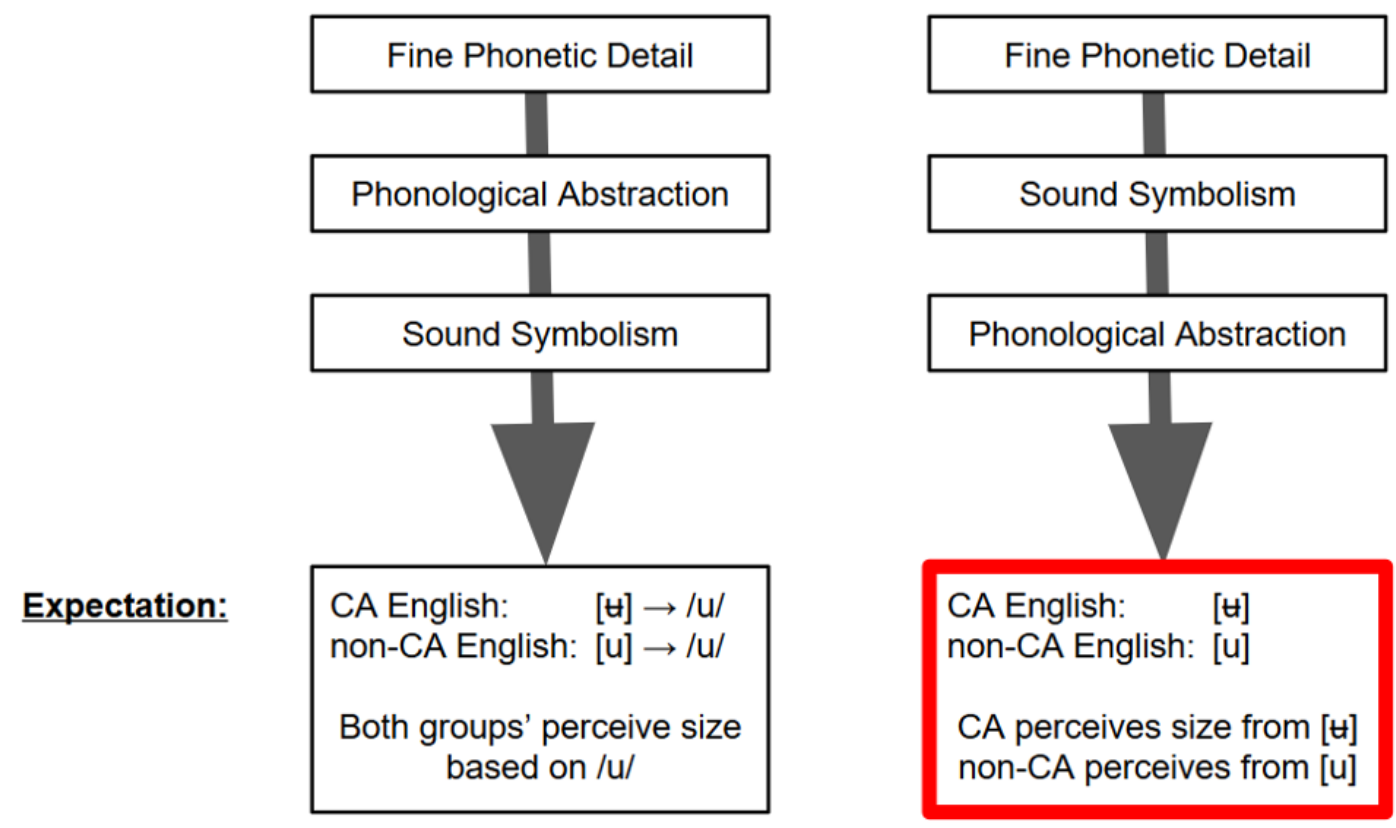

Figure 4. Ordering model for symbolic processing

If sound symbolic perception takes place after phonological abstraction, the CA English $[\mathrm{u}]$ and the non-CA English $[\mathrm{u}]$ would firstly abstract to the phoneme /u/ then both groups would perceive comparable object sizes from $/ \mathrm{u} /$. As such, this ordering would predict no influence of /u/-fronting on perceived size. Because reading task CA English speakers perceived a smaller mean referent object size from $[\mathrm{u}]$ compared to that of non-CA English speakers, this study's results support the second ordering in which a person constructs sound symbolic meaning from fine phonetic information prior to abstracting to a phonological $/ \mathrm{u} /$ representation.

The presence of a dialect effect in the reading task, but not in the listening task suggests that inner speech reflects phonetic properties of a speaker's dialect. Recent fMRI research finds that inner speech produces motor cortex and temporal activity that represents fine articulatory detail consistent with that of overt speech (Kell et al. 2017). Perhaps CA English speakers produce inner speech that exhibits phonetic properties of the CA English dialect such as /u/fronting. Sound symbolic perception may vary in agreement with this 'accented' inner speech.

The results from this study demonstrate that in the absence of overt speech, people incorporate fine phonetic information from their dialect to construct sound symbolic meaning. Future research to build upon the claims laid out in this paper should further investigate dialect features beyond Californian /u/-fronting and semantic domains beyond perceived referent object size. Exploring the interplay between sound symbolic perception and phonetic variation provides insight into how speech identity informs people's representations of objects in the world. 


\section{References}

Bergen, Benjamin. 2004. The psychological reality of phonaesthemes. Language 80(2). 290-311. Bredin, Hugh. 1996. Onomatopoeia as a figure and a linguistic principle. New Literary History 27(3). 555-569.

de Saussure, Ferdinand. 1916. Course in General Linguistics [translated by Wade Baskin, 2011]. London: Duckworth.

Hinton, Leanne; Moonwomon, Birch; Bremner, Sue; Luthin, Herb; Van Clay, Mary; Lerner, Jean; Corcoran, Hazel. 1987. It's not just the Valley Girls: A study of California English. Annual Meeting of the Berkeley Linguistics Society 13. 117-128.

Kell, Christian A.; Darquea, Maritza; Behrens, Marion; Cordani, Lorenzo; Keller, Christian; Fuchs, Susanne. 2017. Phonetic detail and lateralization of reading-related inner speech and of auditory and somatosensory feedback processing during overt reading. Human brain mapping 38(1). 493-508. http://dx.doi.org/10.1002/hbm.23398.

Klink, Richard. 2000. Creating brand names with meaning: The use of sound symbolism. Marketing Letters 11(1). 5-20.

Köhler, Wolfgang. 1929. Gestalt Psychology. New York: Liverlight.

Labov, William; Ash, Sharon; Boberg, Charles. 2006. The Atlas of North American English: Phonetics, Phonology and Sound Change. Berlin: Walter de Gruyter.

Ohala, John. 1997. Sound Symbolism. Proc. $4^{\text {th }}$ Seoul International Conference on Linguistics [SICOL]. 98-103.

Ramachandran, Vilayanur S.; Hubbard, Edward M. 2001. Synaesthesia - a window into perception, thought, and language. Journal of consciousness studies 8(12). 3-34.

Sapir, Edward. 1929. A study in phonetic symbolism. Journal of experimental psychology 12(3). 225-239.

Shinohara, Kazuko; Kawahara, Shigeto. 2010. A cross-linguistic study of sound symbolism: The images of size. Annual Meeting of the Berkeley Linguistics Society 36(1). 396-410. http://dx.doi.org/10.3765/bls.v36i1.3926.

Whalen, Douglas H.; Levitt, Andrea G. 1995. The universality of intrinsic F0 of vowels. Journal of Phonetics 23(3). 349-366. 